\title{
Simple Method to Generate Terawatt-Attosecond X-Ray Free-Electron-Laser Pulses
}

\author{
Eduard Prat ${ }^{*}$ and Sven Reiche \\ Paul Scherrer Institut, CH-5232 Villigen PSI, Switzerland \\ (Received 23 February 2015; published 15 June 2015)
}

\begin{abstract}
X-ray free-electron lasers (XFELs) are cutting-edge research tools that produce almost fully coherent radiation with high power and short-pulse length with applications in multiple science fields. There is a strong demand to achieve even shorter pulses and higher radiation powers than the ones obtained at stateof-the-art XFEL facilities. In this context we propose a novel method to generate terawatt-attosecond XFEL pulses, where an XFEL pulse is pushed through several short good-beam regions of the electron bunch. In addition to the elements of conventional XFEL facilities, the method uses only a multiple-slotted foil and small electron delays between undulator sections. Our scheme is thus simple, compact, and easy to implement both in already operating as well as future XFEL projects. We present numerical simulations that confirm the feasibility and validity of our proposal.
\end{abstract}

DOI: 10.1103/PhysRevLett.114.244801

PACS numbers: 41.60.Cr, 29.20.Ej, 42.55.Vc

X-ray free-electron lasers (XFELs) are modern research tools in various fields such as biology, material science, chemistry, and physics. The existing and planned XFELs are based on the self-amplified spontaneous emission (SASE) mechanism [1,2], and most of them include the option of selfseeding [3-6] to improve the longitudinal coherence of the SASE XFEL. State-of-the-art XFELs are able to produce almost fully coherent radiation with very high pulse energies and very short pulse lengths. For instance, the Linac Coherent Light Source (LCLS) [7] can generate radiation with a pulse energy of the order of $1 \mathrm{~mJ}$ for FWHM pulse lengths of several tens of femtoseconds or more, corresponding to radiation power levels in the gigawatt range. Shorter pulses (e.g., several femtoseconds) can be obtained with pulse energies reduced accordingly. There is, however, a strong demand to achieve even higher pulse powers and/or shorter pulse lengths in research fields such as bioimaging and nonlinear optics — see, for instance, Refs. [8-14].

Several ideas have been proposed to further shorten the pulse length, mostly employing one or more external lasers (see, for example, Refs. [15-21]), but also by generating very short electron bunches [22,23]. Recently, Tanaka proposed a scheme that, in addition to generating shorter XFEL pulses, produces high peak powers up to the terawatt range [24]. His design, however, is complex and imposes significant changes to a standard machine beyond demonstrated beam line elements (like slotted foils [22] and chicane delays): it requires a so-called enhanced-SASE section [17] consisting of an optical laser, an undulator module, and a magnetic chicane, and a section with optical mirrors that delays the radiation beam with respect to the electrons.

Here we propose a novel scheme to generate terawattattosecond XFEL pulses. Our scheme utilizes a baseline configuration of an XFEL facility and adds a slotted foil [22] and very small chicanes to delay the electron bunch between certain undulator modules. Our proposal is simple and compact, and it could be implemented at already existing XFEL facilities, such as LCLS [7] or the Spring-8 Angstrom Compact Free Electron Laser (SACLA) [25], with very small hardware modifications.

The method is based on the superradiant behavior of short pulses [26,27], where the power level can significantly exceed the saturation power of an XFEL while shortening its pulse length. The process starts with several short pulses, which are obtained from a multiple-slotted foil [22] that effectively chops the electron bunch by preserving the emittance only of the bunch slices that travel through the slots. We name the unaffected electron beam region defined by one slot the unspoiled beam region (UBR). Later, by delaying the electron bunch with chicanes, the first radiation short pulse is shifted to fresh parts of the electron bunch, which can further amplify the superradiant spikes. An uneven spacing of the slots prevents the buildup of multiple short pulses and allows the growth of only a single short pulse, where the delays are matched to the spacing of the UBRs.

Figure 1 shows a layout of the proposed scheme and Fig. 2 indicates how the scheme works qualitatively. The first part consists of a slotted foil inserted between the second and third dipole magnet of one of the bunch compressors (BC) of the accelerator. At the slotted foil position there is a strong correlation between the longitudinal and transverse coordinates of the bunch, since at the $\mathrm{BC}$ the dispersion (correlation between transverse and energy coordinates) is high (typically a few $\mathrm{cm}$ ) and, as a requirement for the compression, the beam features a large monotonic correlation between time and energy

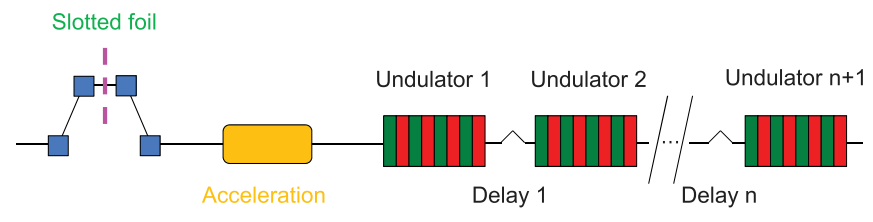

FIG. 1 (color online). Layout of the proposed scheme. 


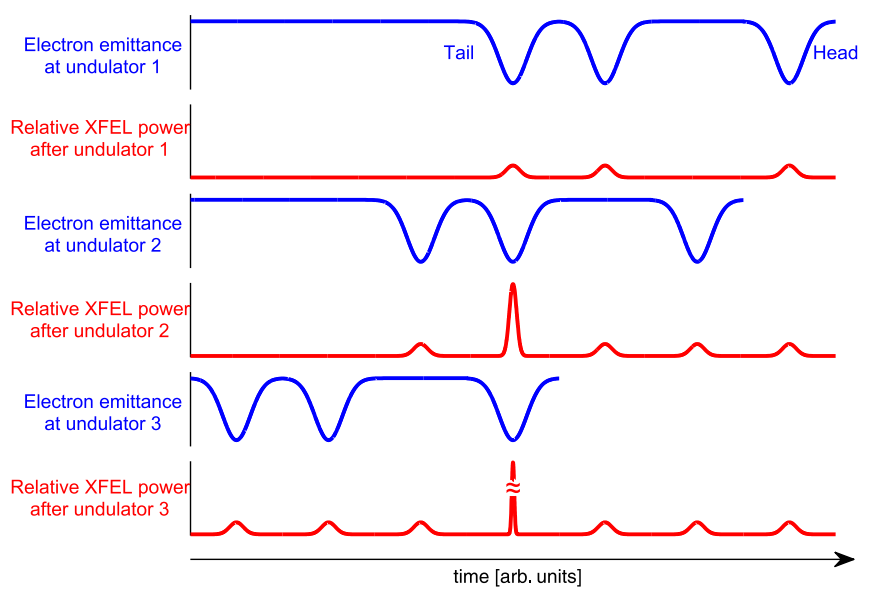

FIG. 2 (color online). Sketch to show the working principle of the proposed scheme for an example case with three undulator sections. The horizontal axis is the same for all the plots: time in the reference system of the radiation field. The electron beam is delayed after each undulator section to overlap all the good emittance regions with the first XFEL subpulse (see text for more details).

(typically of the order of $1 \%$ ). Then, a foil with slots in the transverse bending direction in the $\mathrm{BC}$ can be used to select time slots of the electron beam. The foil contains multiple slots with uneven spacings between them. For instance, the separation between the slots can be $1 x, 2 x, 3 x, 4 x, 5 x$, etc., where $x$ is a certain constant distance. The slotted foil deteriorates the quality of the longitudinal parts of the electron beam that go through the foil-only the electrons passing through the slots will keep the small emittance required to produce XFEL radiation. The slot width cannot be arbitrarily small and has to fulfill certain conditions: the final time duration of the UBR depends on the $\beta$ function, the emittance, and the dispersion at the foil location [22].

After the slotted-foil section the electrons are accelerated to their final energy. The electron bunch then produces normal SASE-XFEL radiation in the first undulator section. An undulator section consists of one or more undulator modules. The output XFEL pulse at the first undulator section consists of several short pulses defined by the UBRs. Following the irregular distribution of the slots, the separation between the different XFEL subpulses is also uneven. After the first undulator section the electrons are delayed in such a way that the second UBR overlaps in the second undulator section with the first radiation subpulse. This causes an efficient seeding of the second UBR with the field from the first part, while the other subpulses, overlapping with electrons with large emittance, will not grow further. This procedure is repeated until all the UBRs have contributed to amplify the first radiation subpulse, while the rest of the radiation pulse, overlapped with bad quality beam slices, is kept to an insignificant power level. A good transverse and longitudinal overlap between the electron and photon beams is required after the chicanes: the transverse overlap can be optimized by tuning the electrons' position and angle using dipole magnet correctors, while the longitudinal overlap can be tuned with the strength of the chicane. In summary, the uneven separation of the slots and the delays of the electron beam are chosen in such a way that only the first subpulse of the XFEL radiation is amplified in all the different stages. At the end a terawatt-attosecond XFEL pulse is produced.

In the first undulator section the XFEL growth does not reach saturation. In the second and subsequent sections the power of the first subpulse goes beyond saturation, but the radiation generated from the rest of the UBR starts from an insignificant level and does not reach saturation. This is to avoid an excessive increase of the energy spread of the electron beam, which would limit the radiation growth of the selected subpulse in the next undulator section. The number of undulator modules per each section is a parameter that needs to be optimized per each case by numerical simulations.

In the second undulator stage the length of the XFEL radiation spikes can be longer or shorter than the length of the UBR, while in the next stages there will be a narrowing of the superradiant spikes. In the case of a longer radiation spike, only one single spike will be enhanced, which will result in a very short radiation pulse; in the other case, several spikes will be created, turning into a longer radiation pulse but also to a higher XFEL power for the same radiation wavelength (since more spikes contribute to the total pulse energy). Some control of choosing the first or the second regime can be obtained by tuning the electron peak current and/or by varying the slot width. In our simulation cases, for a radiation wavelength of $1 \AA$, the pulse will be formed by a few spikes, while for $5 \AA$ only one or two spikes will be created (see below). In the case of more than one spike in a radiation subpulse, the radiation slippage along one undulator section has to be shorter than the separation between the radiation spikes. Otherwise, if the slippage is longer than the spike separation, the spikes would be enhanced by nonfresh parts of the bunch (with increased energy spread due to their contribution to the enhancement of other spikes) and, therefore, the radiation growth would be limited.

In our scheme collective effects like wakefields can be compensated locally [28], in contrast to conventional SASE operation where the entire bunch is supposed to lase, which requires a flat wake potential that can be globally compensated to keep the full bunch in resonance. This opens the way to higher compression factors: the resulting stronger wakefields can be neutralized locally and, in addition, this inhibits the enhancement of competing pulses by the other UBRs.

We have proved the validity of our method by numerical simulations done with the code GENESIS [29]. The calculations are done for radiation wavelengths of 1 and $5 \AA$. The electron beam properties and the lattice parameters have been chosen based on the SwissFEL project [30], presently under construction at the Paul Scherrer Institute in Switzerland. The electron bunch has the following characteristics: a flattop 
longitudinal profile with a peak current of $6 \mathrm{kA}$ and a total charge of $200 \mathrm{pC}$, an energy of $5.8 \mathrm{GeV}$, an energy spread of $350 \mathrm{keV}$, a normalized slice emittance of $300 \mathrm{~nm}$, and an average $\beta$ function along the undulator of $10 \mathrm{~m}$. The assumed emittance value is consistent with our measurements at the SwissFEL Injector Test Facility [31,32]. Each undulator module is $4 \mathrm{~m}$ long and has a period length of $15 \mathrm{~mm}$ and a variable gap with a nominal undulator-parameter value of 1.2. The distance between modules is $0.75 \mathrm{~m}$, which is enough to place a quadrupole magnet to focus the electron beam and a chicane to apply the delays required in our concept. Typical delays are up to several femtoseconds, which can be achieved with $0.5 \mathrm{~m}$ long chicanes consisting of dipole magnets with a length of several centimeters and a standard peak field of about $1 \mathrm{~T}$. The delay is enough to smear out the electron bunching created at the previous undulator section, which helps to have an efficient radiation growth in the next undulator stage.

Figure 3 shows the transverse emittance (for both the horizontal and vertical directions) along the electron bunch at the undulator entrance. The foil has 7 slots that define 7 UBRs along the bunch, which correspond to 7 undulator sections with 6 beam delays between them. Each of the UBRs represents $2.5 \%$ of the total bunch, so $17.5 \%$ of the full bunch will contribute to the generation of the XFEL radiation. The fraction of the electron beam contributing to the lasing process could be increased by using wider slots, but at the expense of longer radiation pulses. The separation between the slots is uneven and increases progressively along the bunch. More UBRs could be obtained for a longer initial bunch, which would require a higher electron bunch charge. We assume that the foil degrades the emittance by a factor of 20 and that there is a smooth transition between the spoiled and unaffected regions of the bunch. Taking into account the considerations of Ref. [22], the realization of this slotted foil would be feasible in the final bunch compressor of a typical XFEL facility.

Based on numerical simulations, we have systematically optimized the number of undulator modules per each section and the linear tapering of the undulator field [33] along the beam line to maximize the output XFEL power

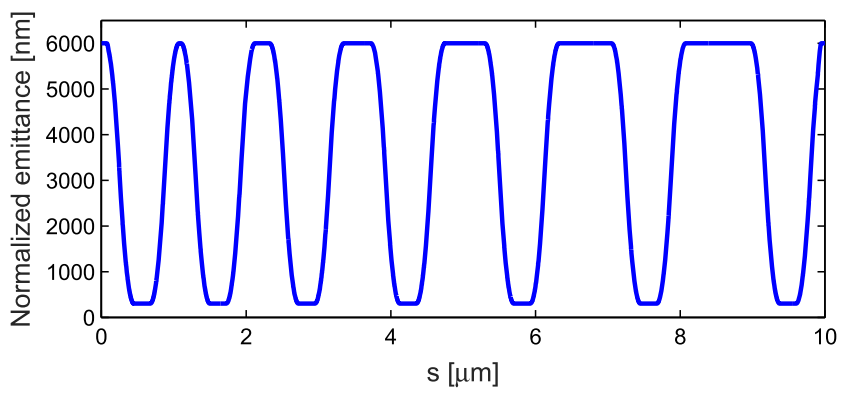

FIG. 3 (color online). Normalized horizontal and vertical emittance along the longitudinal position of the electron bunch used in the numerical simulations. and energy. Tapering is necessary to compensate the energy loss of the electrons as they generate XFEL radiation along the undulator beam line, thus allowing the radiation field growth above the saturation level. We have run 5 simulations using different seeds for the generation of the shot noise of the electron beam. For the $1 \AA$ case, the best configuration is with four modules in the first two undulator sections and with two modules in the rest of the sections. In total, 18 undulator modules are used. The applied delays between the undulator sections to match all the UBRs with the first radiation subpulse are the following (written in consecutive order): 2.7, 3.6, 4.5, 5.0, 5.7, and 6.1 fs. We note that more undulator modules could be placed in the last undulator section to further increase the XFEL power until the spikes slip out of the last UBR. Figure 4 shows the taper optimization and the radiation pulse energy along the undulator line for the best case. The optimum taper is $0.12 \%$, which corresponds to a final XFEL pulse energy of $180 \pm 14 \mu \mathrm{J}$ (the error bars indicate the variation among the five simulated seeds). For these optimum conditions, the maximum XFEL power along the pulse is $1.07 \pm 0.24 \mathrm{TW}$ and the rms pulse length is $234 \pm 15$ as. For three of the five simulated cases the FWHM pulse length is below 45 as, because a single spike is dominant. However, for our conditions it is impossible to enforce such singles spikes at $1 \AA$ due to the stochastic nature of the generation of SASE spikes.

We have also simulated the performance of our scheme for a radiation wavelength of $5 \AA$. The optimum
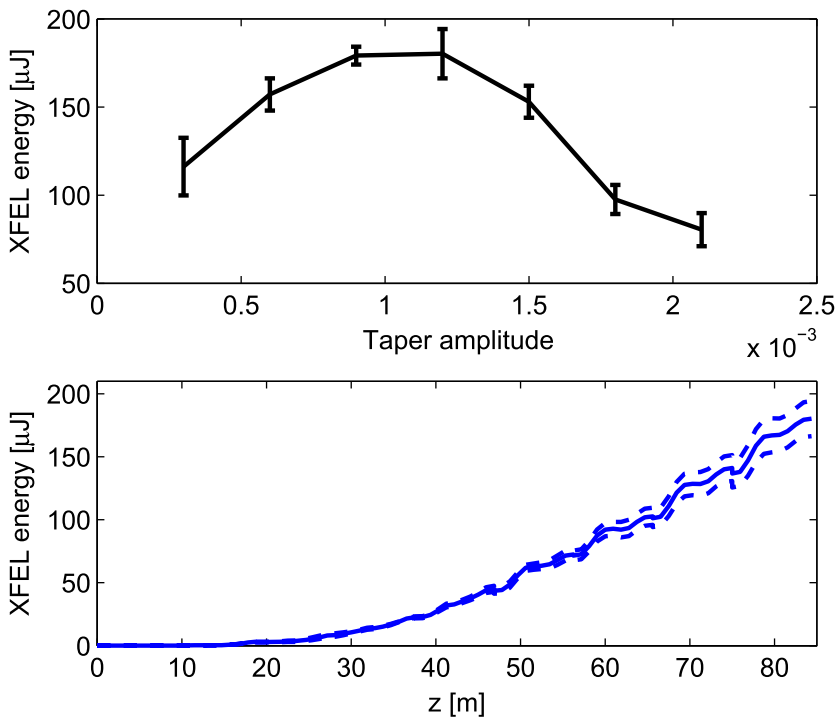

FIG. 4 (color online). XFEL energy versus undulator tapering (upper plot) and XFEL energy for the optimum taper along the undulator beam line (lower plot). The solid line of the lower plot indicates the mean pulse energy of the 5 simulated seeds, while the dashed lines include the error bars considering the standard variation over the 5 seeds. The final energy is $180 \pm 14 \mu \mathrm{J}$ and is obtained for a linear tapering of $0.12 \%$. 

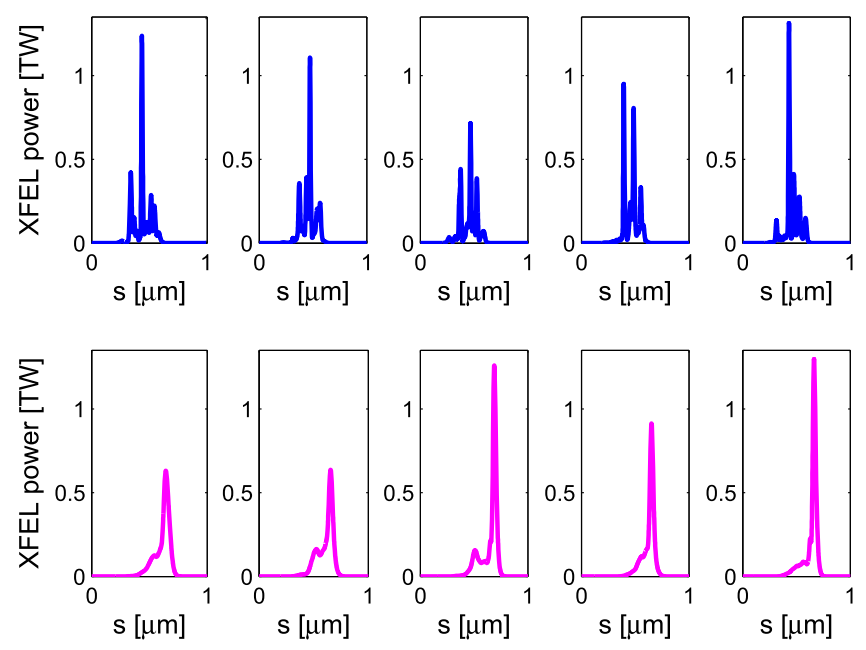

FIG. 5 (color online). XFEL radiation profiles for the different simulations. The blue curves correspond to the simulations at a radiation wavelength of $1 \AA$ while the magenta curves are for $5 \AA$. The horizontal and vertical scales are the same for all the plots.

configuration for $5 \AA$ is with two undulator modules in the first and last sections and with one module in the rest of the undulator sections, which results in a total of 9 undulator modules. The best linear taper for this case is $0.13 \%$. The electron delays between the undulator sections are similar to the $1 \AA$ case. The obtained XFEL energy is $206 \pm 24 \mu \mathrm{J}$, the maximum XFEL power $0.95 \pm 0.32 \mathrm{TW}$, and the rms and FWHM pulse lengths are $222 \pm 28$ and $142 \pm 37$ as, respectively.

Figure 5 shows the XFEL radiation pulse at the end of the undulator line for all the simulations. For the radiation wavelength of $5 \AA$, due to the longer slippage, the final XFEL pulse consists of almost one single spike. Figure 6 shows the maximum XFEL power along the undulator line for all simulated cases. A power of $\approx 1 \mathrm{TW}$ is reached in all simulations. For the $5 \AA$ cases, however, only half of the undulator length is necessary to reach this power level.

The bunch length jitter coming from amplitude and phase jitter of the accelerator rf structures can limit the performance of our procedure: a significant variation of the bunch length may compromise a good longitudinal overlap between the electron and photon beams required after the chicanes. In our case, the bunch length jitter is expected to be less than $10 \%$ [34], which should not significantly affect the performance of the scheme. For more unstable conditions, taking longer UBRs will improve the robustness of the method.

In conclusion, we have presented a novel method to generate terawatt-attosecond XFEL pulses, which is simple, compact, and easy to implement. In addition to standard components, it only needs a multiple-slotted foil and small electron beam delays between undulator sections. It does not require any external laser or seeding scheme. Our scheme could be easily tested in any of the present or future XFEL facilities worldwide. The immediate implementation of our proposal at facilities such as LCLS or

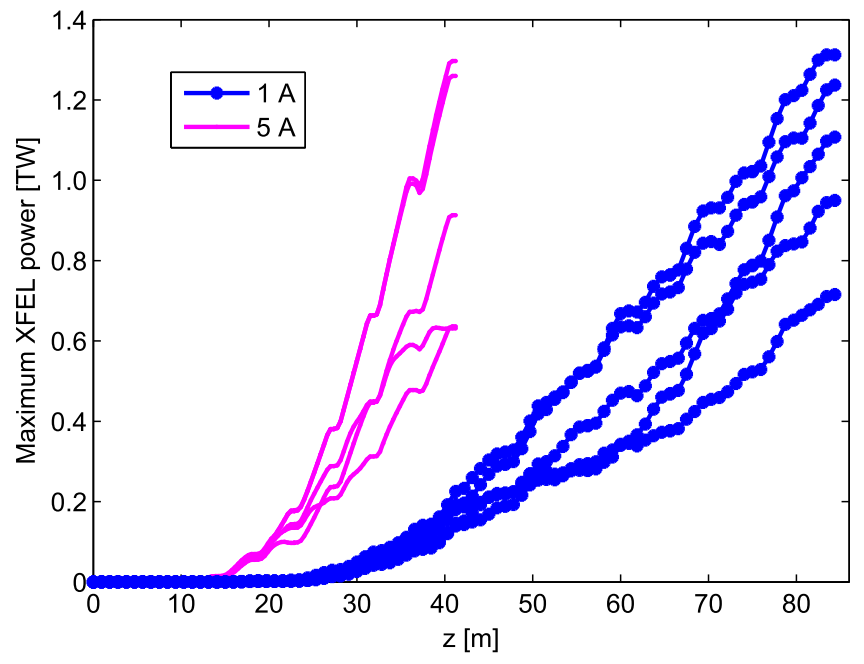

FIG. 6 (color online). Maximum XFEL power along the undulator line for the different simulated cases.

SACLA would only require the installation of a multipleslotted foil and small chicane delays between certain undulator modules. We have shown with numerical simulations that this proposal improves the radiation pulse power and length by orders of magnitude with respect to the state-of-the-art XFELs: for SwissFEL parameters, radiation pulses of about 1 TW and rms lengths of about 200 as can be generated in about $80 \mathrm{~m}$ of undulator line for a wavelength of $1 \AA$, and in about $40 \mathrm{~m}$ for a wavelength of $5 \AA$. We note that our scheme offers scalability towards higher power by using longer pulses and more slots. The system can also provide higher pulse energies by increasing the width of the slots. This would, however, increase the pulse length toward the femtosecond level but make it also applicable for soft XFELs, where the minimum pulse length is determined by the longer cooperation length. The XFEL pulses obtained from our scheme pave the way for outstanding progress in research fields like nonlinear optics and bioimaging, entering the regime required for single-molecule imaging experiments [9].

We would like to thank Thomas Schietinger, Hans Braun, and Terry Garvey for fruitful discussions and careful proofreading of the manuscript. We acknowledge Bruce Patterson, Martin Beye, and Nina Rohringer for their input on the XFEL user requirements.

*eduard.prat@psi.ch

[1] A. M. Kondratenko and E. L. Saldin, Part. Accel. 10, 207 (1980).

[2] R. Bonifacio, C. Pellegrini, and L. M. Narducci, Opt. Commun. 50, 373 (1984).

[3] J. Feldhaus, E. L. Saldin, J. R. Schneider, E. A. Schneidmiller, and M. V. Yurkov, Opt. Commun. 140, 341 (1997). 
[4] E. L. Saldin, E. A. Schneidmiller, Yu. V. Shvyd'ko, and M. V. Yurkov, Nucl. Instrum. Methods Phys. Res., Sect. A 475, 357 (2001).

[5] G. Geloni, V. Kocharyan, and E. Saldin, DESY Report No. 10-053, 2010.

[6] P. Emma et al., Nat. Photonics 6, 693 (2012).

[7] P. Emma et al., Nat. Photonics 4, 641 (2010).

[8] F. Capotondi, M. Dell'Angela, M. Malvestuto, and F. Parmigiani, in Synchrotron Radiation. Basics, Methods and Applications, edited by S. Mobilio, F. Boscherini, and C. Meneghini (Springer, Berlin, 2015), Chap. 30, p. 761 .

[9] A. Fratalocchi and G. Ruocco, Phys. Rev. Lett. 106, 105504 (2011).

[10] N. Rohringer et al., Nature (London) 481, 488 (2012).

[11] M. Beye, S. Schreck, F. Sorgenfrei, C. Trabant, N. Pontius, C. Schüßler-Langeheine, W. Wurth, and A. Föhlisch, Nature (London) 501, 191 (2013).

[12] S. Schreck et al., Phys. Rev. Lett. 113, 153002 (2014).

[13] H. Yoneda, Y. Inubushi, M. Yabashi, T. Katayama, T. Ishikawa, H. Ohashi, H. Yumoto, K. Yamauchi, H. Mimura, and H. Kitamura, Nat. Commun. 5, 5080 (2014).

[14] R. Neutze, R. Wouts, D. van der Spoel, E. Weckert, and J. Hajdu, Nature (London) 406, 752 (2000).

[15] E. L. Saldin, E. A. Schneidmiller, and M. V. Yurkov, Opt. Commun. 239, 161 (2004).

[16] A. A. Zholents and W. M. Fawley, Phys. Rev. Lett. 92, 224801 (2004).

[17] A. A. Zholents, Phys. Rev. ST Accel. Beams 8, 040701 (2005).

[18] A. A. Zholents and G. Penn, Phys. Rev. ST Accel. Beams 8, 050704 (2005).
[19] E. L. Saldin, E. A. Schneidmiller, and M. V. Yurkov, Phys. Rev. ST Accel. Beams 9, 050702 (2006).

[20] A. A. Zholents and M.S. Zolotorev, New J. Phys. 10, 025005 (2008).

[21] D. Xiang, Z. Huang, and G. Stupakov, Phys. Rev. ST Accel. Beams 12, 060701 (2009).

[22] P. Emma, K. Bane, M. Cornacchia, Z. Huang, H. Schlarb, G. Stupakov, and D. Walz, Phys. Rev. Lett. 92, 074801 (2004).

[23] S. Reiche, P. Musumeci, C. Pellegrini, and J. B. Rosenzweig, Nucl. Instrum. Methods Phys. Res., Sect. A 593, 45 (2008).

[24] T. Tanaka, Phys. Rev. Lett. 110, 084801 (2013).

[25] T. Ishikawa et al., Nat. Photonics 6, 540 (2012).

[26] R. Bonifacio, L. De Salvo Souza, P. Pierini, and N. Piovella, Nucl. Instrum. Methods Phys. Res., Sect. A 296, 358 (1990).

[27] R. Bonifacio, N. Piovella, and B. W. J. McNeil, Phys. Rev. A 44, R3441 (1991).

[28] S. Reiche, P. Emma, and C. Pellegrini, Nucl. Instrum. Methods Phys. Res., Sect. A 507, 426 (2003).

[29] S. Reiche, Nucl. Instrum. Methods Phys. Res., Sect. A 429, 243 (1999).

[30] PSI Report No. 10-04, 2012, edited by R. Ganter.

[31] PSI Report No 10-05, 2010, edited by M. Pedrozzi.

[32] E. Prat, M. Aiba, S. Bettoni, B. Beutner, S. Reiche, and T. Schietinger, Phys. Rev. ST Accel. Beams 17, 104401 (2014).

[33] N. M. Kroll, P. L. Morton, and M. Rosenbluth, IEEE J. Quantum Electron. 17, 1436 (1981).

[34] B. Beutner, in Proceedings of the 34th International FreeElectron Laser Conference, Nara, Japan, 2012 (JACoW, Geneva, 2013), p. 297. 